\title{
Dual role of MdSND1 in the biosynthesis of lignin and in signal transduction in response to salt and osmotic stress in apple
}

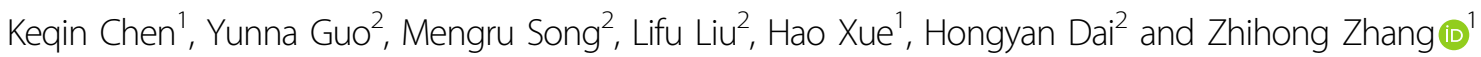

\begin{abstract}
Clarifying the stress signal transduction pathway would be helpful for understanding the abiotic stress resistance mechanism in apple (Malus $\times$ domestica Borkh.) and could assist in the development of new varieties with high stress tolerance by genetic engineering. The key NAC transcription factor SND1, which is involved in the lignin biosynthesis process in apple, was functionally analyzed. The results of the stress treatments indicated that MdSND1 could be induced by salt, mannitol and ABA. Compared with wild-type GL-3 plants, MdSND1-overexpressing apple plants with greater antioxidant capacity and lignin were more resistant to salt and simulated osmotic stress, while RNAi plants were more vulnerable. Additionally, molecular experiments confirmed that MdSND1 could regulate the biosynthesis of lignin by activating the transcription of MdMYB46/83. Moreover, genes known to be involved in the stress signal transduction pathway (MdAREB1A, MdAREB1B, MdDREB2A, MdRD29A, and MdRD22) were screened for their close correlations with the expression of MdSND1 and the response to salt and osmotic stress. Multiple verification tests further demonstrated that MdSND1 could directly bind to these gene promoters and activate their transcription. The above results revealed that MdSND1 is directly involved in the regulation of lignin biosynthesis and the signal transduction pathway involved in the response to both salt and osmotic stress in apple.
\end{abstract}

\section{Introduction}

Plants live in constantly changing environments, and stressful conditions can limit their growth, development, and propagation ${ }^{1}$ or even cause adaptive changes in their morphology and biological processes. Generally, stressful conditions induce plant responses not only at the physiological and biochemical levels but also at the cellular and molecular levels ${ }^{2}$. During the molecular process underlying stress signal perception to stress-responsive gene expression, many transcription factors are involved and play roles in signal transduction ${ }^{3}$. Understanding and clarifying stress signaling and responses would be helpful for explaining the stress resistance mechanism in plants

Correspondence: Hongyan Dai (daihy@syau.edu.cn) or

Zhihong Zhang (zhangz@syau.edu.cn)

${ }^{1}$ Group of Molecular Biology of Fruit Trees, College of Horticulture, Shenyang Agricultural University, 120 Dongling Road, Shenyang, Liaoning 110866, China ${ }^{2}$ Group of Fruit Germplasm Evaluation \& Utilization, College of Horticulture, Shenyang Agricultural University, 120 Dongling Road, Shenyang, Liaoning 110866, China and would increase our ability to enhance the stress tolerance of crops to achieve agricultural sustainability and food security for an increasing global population ${ }^{1}$.

The NAC (NAM, no apical meristem; ATAF, Arabidopsis transcriptional activator and CUC, cup-shaped cotyledon) proteins, which have a highly conserved DNAbinding domain in their $\mathrm{N}$-terminal region and variable domains in their C-terminal region, usually function as transcription factors in plants and play important roles in plant growth and development ${ }^{4-11}$. Secondary wallassociated NAC domain protein 1 (SND1), a transcription factor that functions upstream of MYB46, SND3, MYB103, and $\mathrm{KNAT7}^{12}$, is expressed specifically in interfibers and lignocellulosic fibers in plant stems and plays an important role in fiber thickening ${ }^{13}$. Simultaneous knockout of SND1/NST3 and NST1 resulted in severely reduced expression of secondary wall biosynthesis genes in conjunction with loss of all three major secondary wall components: cellulose, xylan and 
lignin ${ }^{10,14}$. In addition, NAC transcription factors have also been reported to be involved in the regulation of signal transduction and plant responses to various biotic and abiotic stresses ${ }^{15-18}$.

Drought and high salinity are common abiotic stresses that have an adverse effects on plant growth and productivity and can lead to deterioration of fruit quality ${ }^{19,20}$. Early perception of the transduction of abiotic stress signals in plants is primarily controlled by several transcription factors ${ }^{21,22}$ that can directly or indirectly regulate plant responses ${ }^{20,23-25}$. Dehydration response element-binding protein 2 (DREB2) plays an important role in the response to abiotic stress, and many DREB2 homologs have been reported to be induced by salt or drought stress, including AtDREB2A, AtDREB2B, and AtDREB2C in Arabidopsis; ${ }^{26,27}$ GmDREB2 in Glycine max; $;^{28}$ PeDREB2 in Populus euphratica; $;^{29}$ OsDREB2A in Oryza sativa; ${ }^{30}$ EsDREB2B in Eremosparton songoricum; ${ }^{31}$ and SIDREB2 in Solanum lycopersicum ${ }^{32}$. Abscisic acidresponsive element-binding protein 1 (AREB1) (also named ABF2) belongs to the basic leucine zipper (bZIP) transcription factor family and can regulate the expression of ABA-inducible genes by binding to the ABAresponsive element (ABRE) motif in their promoter. The expression of $A R E B 1$ is upregulated not only by $\mathrm{ABA}$ but also in response to drought and high-salinity stress ${ }^{33,34}$. AREB1/ABF2 has been reported to function predominantly in regulating the expression of genes whose products function downstream of SnRK2 kinases in the ABA signaling pathway in response to osmotic stress ${ }^{33}$. Several stress-responsive genes, such as RD22 and RD29, are induced in response to osmotic stress through AREB transcription factors in an ABA-dependent manner ${ }^{34,35}$.

Apple plants are widely distributed worldwide, and apple fruits have high nutritional value. Both drought and salt stress are two environmental factors limiting apple growth in some areas. Previous studies have found that the lignin biosynthesis pathway in apple is closely related to the signaling pathways of stress responses. MdMYB46, a key regulator of secondary cell wall formation, can enhance the stress tolerance of apple by directly activating stress-responsive signals ${ }^{36}$. Whether other types of transcription factors involved in lignin metabolism (such as NACs) have similar functions has become an interesting question for future studies. In this study, we found that, in addition to regulating the accumulation of lignin in apple plants, MdSND1 also participates in the regulation of the stress signal transduction pathway by activating the expression of stress-responsive genes.

\section{Results}

MdSND1 has conserved NAC DNA-binding regions

Overexpression of AtSND1 can increase secondary cell wall thickening in Arabidopsis ${ }^{9}$. To identify SND1 in apple, we first used the AtSND1 amino acid sequence to search for orthologs in the apple genome (GDDH13 V1.1 database). Phylogenetic analysis of apple SND1 candidate proteins (EgWND1, MtNST1, OsSWNs, ZmSWNs, BdSWNs, PtrNACs, and PtrWNDs) and Arabidopsis secondary cell wall NAC proteins was then carried out (Fig. 1A). The phylogenetic tree showed that both MD06G1121400 and MD14G1137900 were homologous with AtSND1, but the sequence similarity results showed that MD06G1121400 was more similar to AtSND1 (Fig. S1); therefore, MD06G1121400 was named MdSND1 in our study. The amino acid sequences of MD06G1121400, MD14G1137900, AtSND1, PtrWND1A, and PtrWND1B were also compared, and the highly conserved DNAbinding domain (indicated in the red box in the figure) was identified in all of their $\mathrm{N}$-terminal sequences (Fig. 1B).

\section{MdSND1 functions in the nucleus and is sensitive to stress signals}

For subcellular localization, a pRI-MdSND1-eGFP vector was constructed on the basis of a pRI-eGFP vector and contained the MdSND1 coding sequence region (without the stop codon). The fusion vector was then transiently introduced into Nicotiana benthamiana leaves via Agrobacterium-mediated infection. As shown in Fig. 2A, the GFP fluorescence signal revealed that MdSND1 was localized to the nucleus. Moreover, the transcriptional activation region of MdSND1 was found to be present in the C-terminal (Fig. 2B, C) by the use of a yeast twohybrid system.

Compared with wild-type seedlings, AtSND1 deletion mutant seedlings with reduced anthocyanin contents are more sensitive to salt stress ${ }^{13}$. To determine the sensitivity of MdSND1 to abiotic stress, GL-3 apple plants were treated with exogenous $\mathrm{ABA}, \mathrm{NaCl}$, and mannitol. It was found that the expression levels of MdSND1 were significantly upregulated under these stresses (Fig. 2D).

\section{MdSND1 positively regulates salt and simulated drought stress tolerance in apple}

MdSND1 overexpression and RNAi vectors with the CaMV 35S fragment in the promoter were transformed into GL-3 plantlets (Fig. S2A), and three MdSND1-overexpressing transgenic lines, namely, OE-MdSND1-5, OE-MdSND1-7, and OE-MdSND1-16, and five MdSND1-RNAi lines of GL-3 apple, namely, RNAi-MdSND1-1, RNAi-MdSND1-3, RNAiMdSND1-10, RNAi-MdSND1-12, and RNAi-MdSND1-17, were obtained. The transcript levels of MdSND1 in the three overexpressing apple lines significantly increased (Fig. S2B), while those in the MdSND1-RNAi lines significantly decreased (Fig. S2C).

The OE-MdSND1-5, OE-MdSND1-16, RNAi-MdSND1-1, and RNAi-MdSND1-10 transgenic apple lines and wild-type apple plants (GL-3) were subjected to long-term stress 


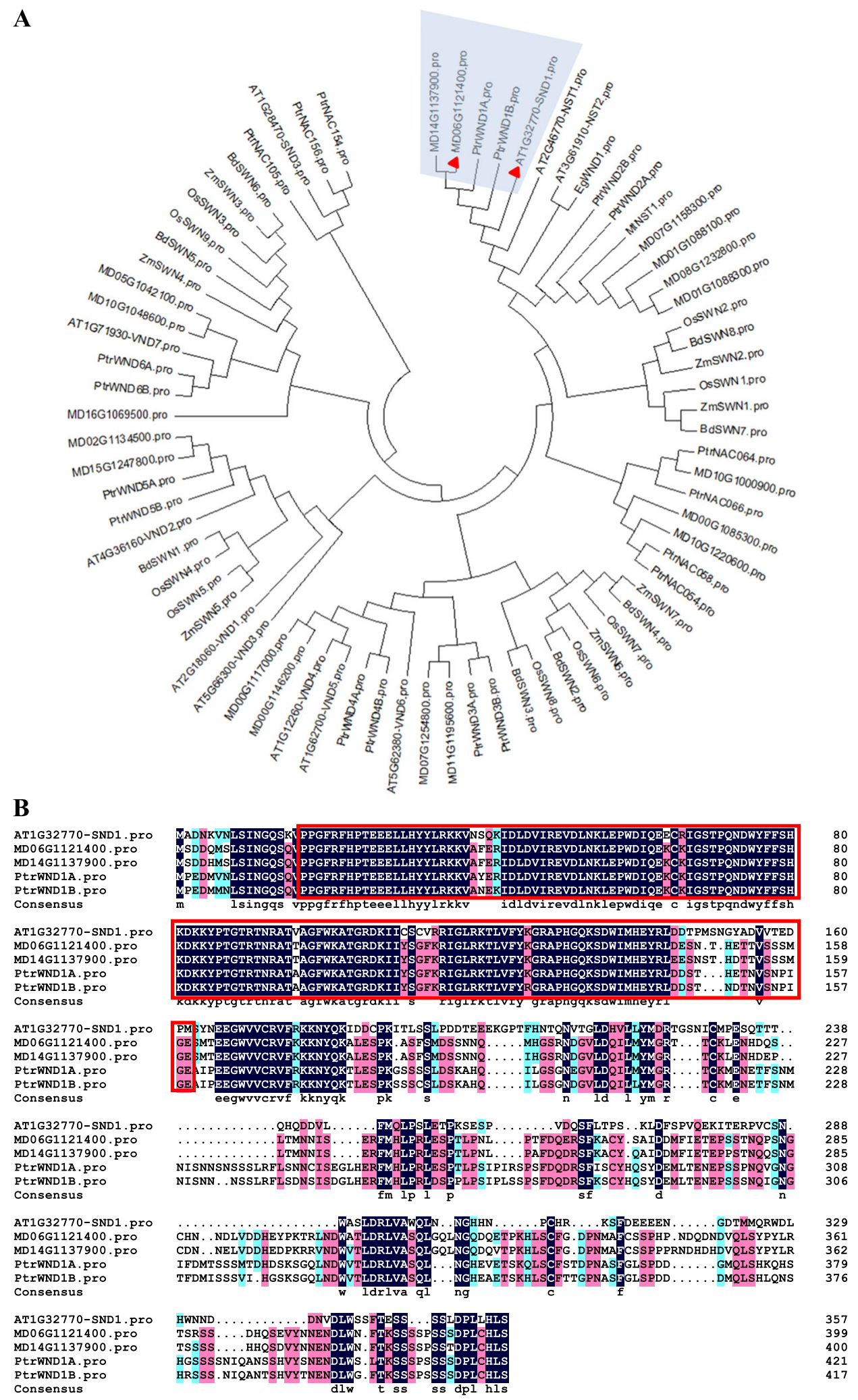

Fig. 1 Phylogenetic analysis and sequence alignment of MdSND1. A Phylogenetic analysis of AtSND1 orthologs. AT (Arabidopsis thaliana); Mt (Medicago truncatula); Eg (Eucalyptus grandis); Os (Oryza sativa); Bd (Brachypodium distachyon); Zm (Zea mays); Ptr (Populus trichocarpa); MD (Malus domestica). B Multiple sequence alignment of AtSND1, MdSND1, and PtrWND1A/1B. The NAC DNA-binding functional regions are marked in the red box 
A
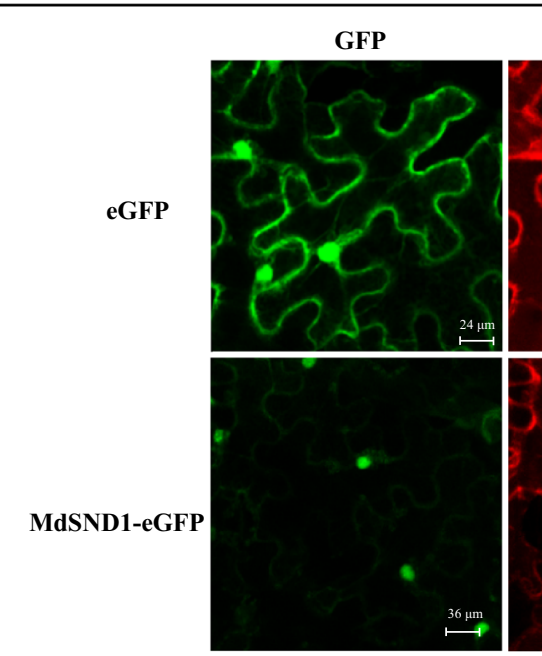

B

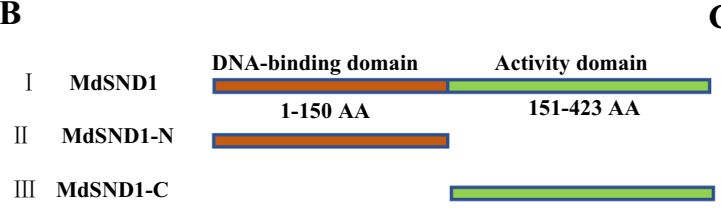

C

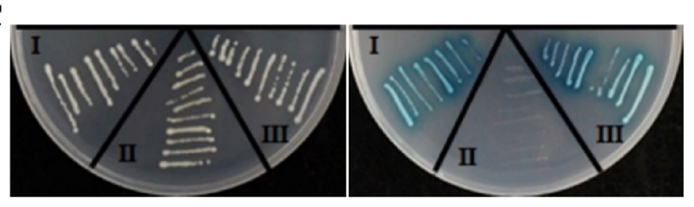

$-\mathbf{T}$

-T/-H/-A/+x-alpha-gal

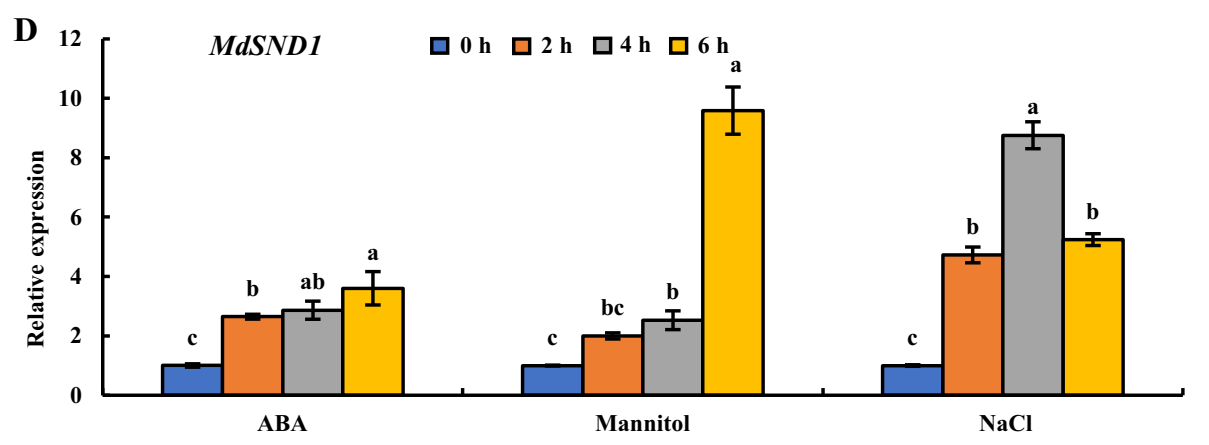

Fig. 2 Subcellular localization, transcriptional activation, and stress induction of MdSND1 in apple. A Subcellular localization of pRI-MdSND1eGFP in epidermal cells of tobacco leaves. B Amino acid sequence structure of full-length MdSND1, the MdSND1 N-terminal region and the MdSND1 C-terminal region. C Transcriptional activation assays of MdSND1. The GAL4 DNA-binding domain (GAL4DB) was fused to the MdSND1 N-terminal region, MdSND1 C-terminal region, and full-length MdSND1 sequence, after which the constructs were transferred into yeast. $(-\mathrm{T} /-\mathrm{H} /-\mathrm{A}+\mathrm{X}$-alphagal) means selective medium supplemented with 5-bromo-4-chloro-3-indolyl-alpha-D-galactoside but lacking Trp, His, and adenine, while (-T) indicates selective medium lacking Trp. D MdSND1 was induced by osmotic stress. The error bars indicate the standard deviations (SDs) of three biological replicates. The letters indicate the level of significance $(P<0.05$, according to Duncan's multiple range test)

treatments to explore the function of MdSND1 in apple. The apple plants showed different phenotypes in response to abiotic stress. Under the salt treatment, the leaves of the $M d S N D 1-R N A i$ apple plants were yellowish-brown and severely curled, while those of the MdSND1-overexpressing plants were still green, comparable to those of the wild-type plants (red circle in Fig. 3A). Similarly, there were no obvious phenotypic changes in the leaves of the MdSND1-overexpressing plants under mannitol stress treatment, while the leaves of the MdSND1-RNAi plants showed a large area of browning. Based on the above description, the MdSND1overexpressing apple plants appeared to be more resistant to both salt and osmotic stress than the wild-type plants did, while the MdSND1-RNAi plants were more vulnerable.

Reactive oxygen species (ROS) act as important molecules during plant stress responses and play a key role in activating downstream metabolic pathways. Since 3,3'diaminobenzidine (DAB) and nitro blue tetrazolium (NBT) can produce reddish-brown and blue precipitates, respectively, after reacting with ROS, they were used to detect $\mathrm{H}_{2} \mathrm{O}_{2}$ and superoxide $\left(\mathrm{O}_{2}{ }^{-}\right)$anions in the leaves of apple plants after stress treatments. The DAB and NBT staining results showed that there were lower levels of ROS in the leaves of $M d S N D 1$-overexpressing apple 

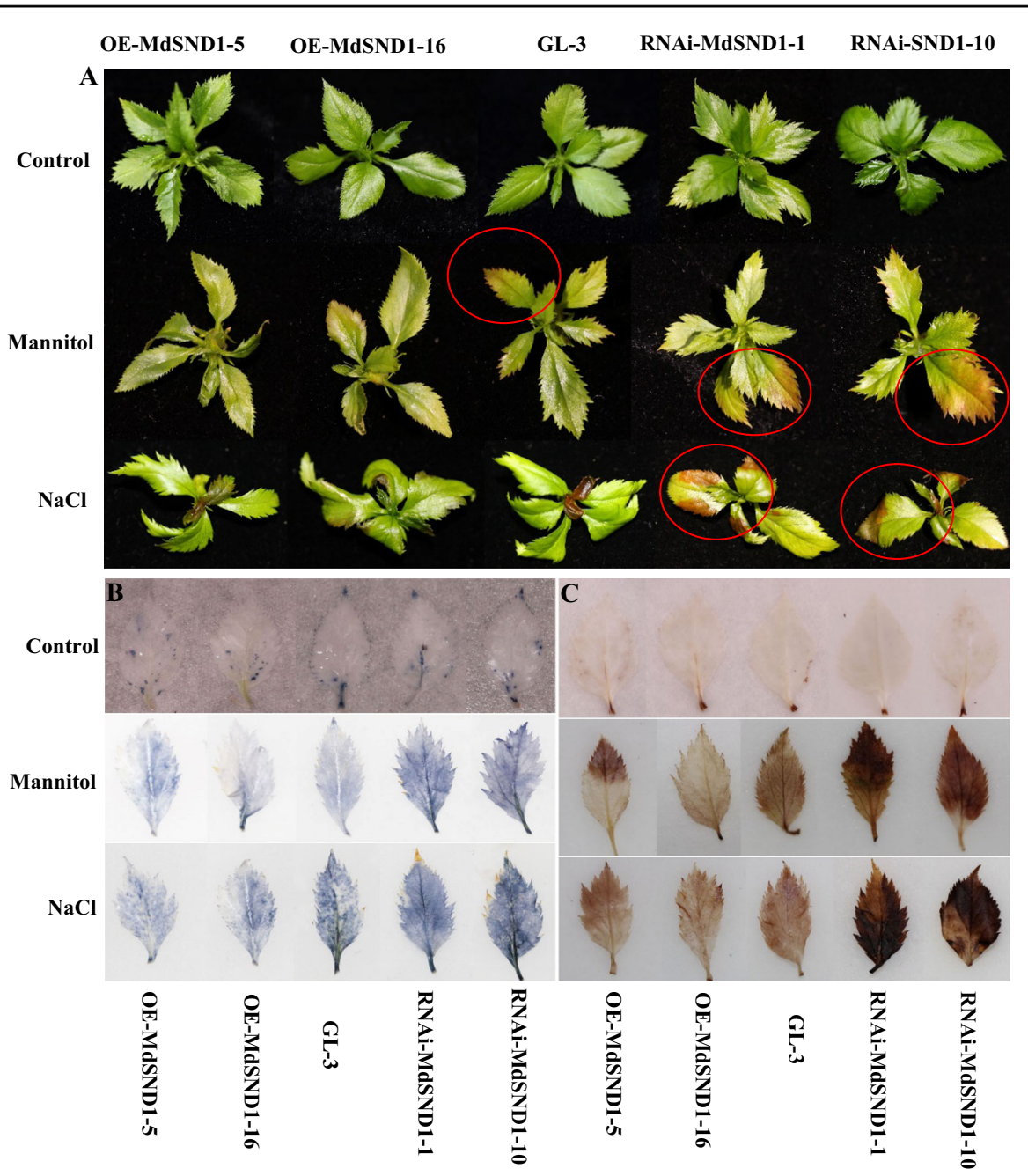

Fig. 3 MdSND1 enhanced the tolerance of transgenic apple plants to salt and osmotic stress. A MdSND1-overexpressing and MdSND1-RNAi apple plants showed different phenotypes under salt and osmotic stresses. Two hundred millimolar $\mathrm{NaCl}$ or $300 \mathrm{mM}$ mannitol was added to the medium when the nontransgenic GL-3 apple plants and transgenic apple were twenty days old. B Detection of ROS levels in apple leaves of plants under salt and mannitol treatment for 10 days according to nitro blue tetrazolium (NBT) staining. The darker the blue precipitate is, the higher the ROS level. C Detection of ROS levels in apple leaves of plants under salt and mannitol treatment for 10 days according to 3,3'-diaminobenzidine (DAB) staining. The darker the brown precipitate is, the higher the ROS level

plants compared with the nontransgenic plants, while there were greater levels in the MdSND1-RNAi plants (Fig. 3B, C).

The contents of stress-related metabolites in apple plants under both salt and osmotic stress are presented in Fig. 4 . Like the results of the DAB/NBT staining showed, the content of $\mathrm{H}_{2} \mathrm{O}_{2}$ in the leaves of the $M d S N D 1$-overexpressing apple plants was lower than that in the nontransgenic plants, while it was the highest in the MdSND1-RNAi plants (Fig. 4A). The relative water content of the leaves is a potential indicator of plant water loss under abiotic stress. The relative water content of the MdSND1-overexpressing apple plants was the highest after exposure to salt and osmotic stress (Fig. 4B). The proline content was positively correlated with stress resistance; proline plays a role in preventing cell dehydration. Compared with that in wild-type GL-3 plants, the proline content in the MdSND1overexpressing apple plants was highest, while it was lowest in the RNAi plants (Fig. 4C). MDA is a product of plant membrane lipid peroxidation and indicates the degree of cell membrane lipid peroxidation. Under abiotic stress, there was less MDA accumulation in the $M d S N D 1$-overexpressing apple leaves than in the wild-type GL-3, while there was more MDA accumulation in the RNAi plants (Fig. 4D).

The transcript level of the secondary cell wall formation key regulatory genes MdMYB46/83 in apple are positively regulated by MdSND1

Since the lignin content showed a strong correlation with the transcript level of MdSND1 (Fig. S3A), the effect of 

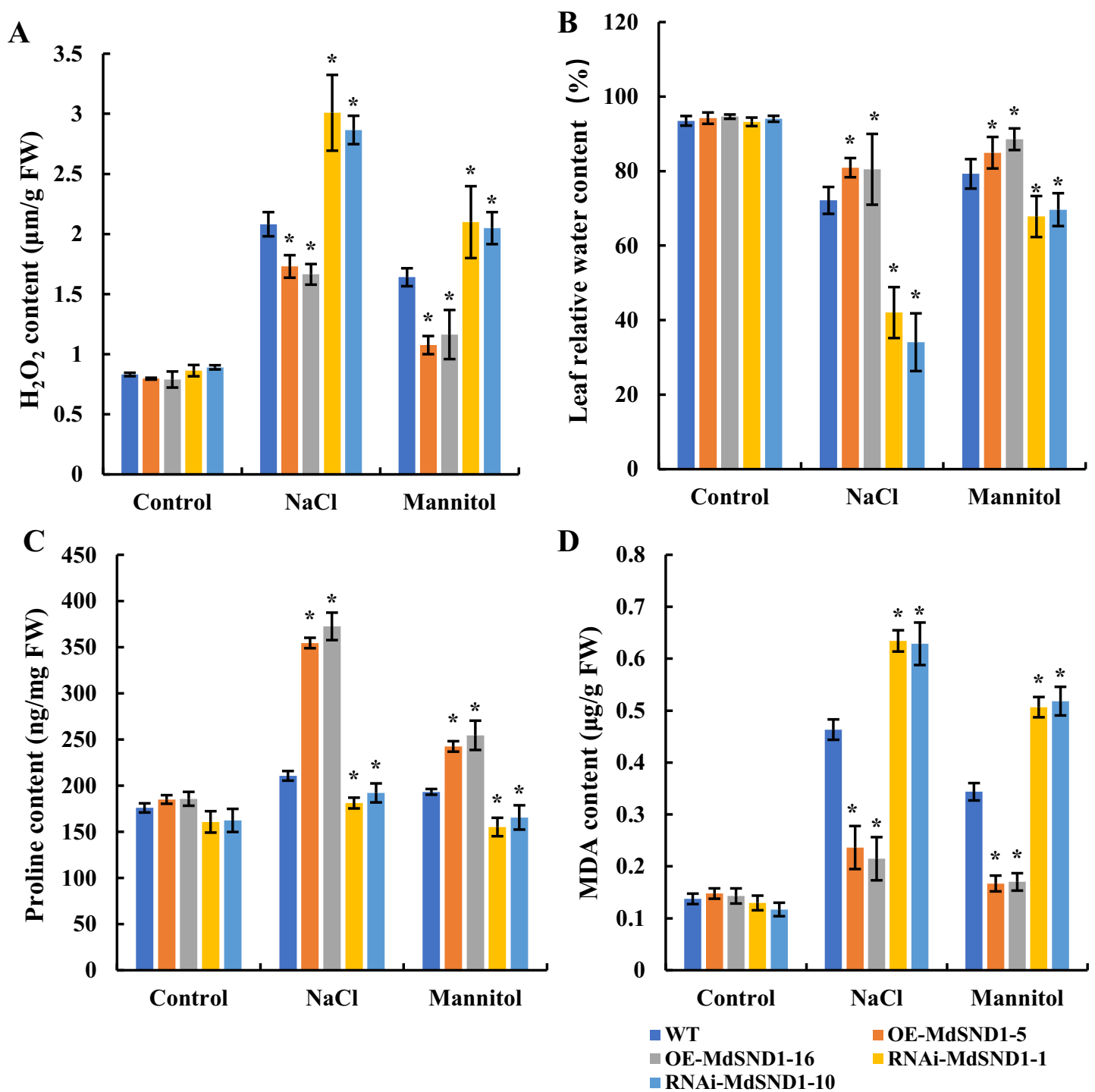

Fig. 4 Comparison of the content of stress-related metabolites in transgenic apple plants under salt and osmotic stress. $\mathbf{A}$ Content of $\mathrm{H}_{2} \mathrm{O}_{2}$. B Relative water content. C Content of proline. D Content of malondialdehyde (MDA). The error bars indicate the standard deviations (SDs) of three biological replicates, ${ }^{*} P<0.05$ (according to $t$-tests)

MdSND1 on lignin metabolism was investigated. The expression levels of lignin biosynthesis-related MYB transcription factors and structural genes in transgenic apple plants were examined. The transcript levels of lignin biosynthesis genes (MdCCR, MdCOMT, MdHCT, MdF5H, $M d 4 C L, M d C A D, M d C 3 H$, and $M d C 4 H)$ and MYB genes $(M d M Y B 46, M d M Y B 83 A$, and $M d M Y B 83 B)$ were significantly upregulated in the $M d S N D 1$-overexpressing lines but were downregulated in the RNAi lines (Fig. S3B, C).

Previous studies have shown that MYB83 and MYB46 redundantly regulate secondary wall formation in fibers and vessels and are direct targets of SND $1^{37}$. To clarify the regulatory role of MdSND1 in the formation of secondary cell walls, we analyzed the promoter region of the MdMYB46 and MdMYB83 genes.

SND1 can bind specifically to secondary wall NACbinding element (SNBE) sites in its downstream gene promoter $^{9,10}$. Multiple SND1-binding sites were found in the promoters of the lignin biosynthesis regulatory genes (MdMYB46, $M d M Y B 83 A$, and $M d M Y B 83 B$ ) (Fig. 5A). To investigate whether MdSND1 binds to the MdMYB46 and MdMYB83 promoters, we applied EMSAs that involved a GST-SND1 fusion protein and an SNBE promoter fragment (Fig. 5B). It was found that the recombinant MdSND1 protein was able to bind to the $M d M Y B 46$ and $M d M Y B 83$ promoter fragments, causing 


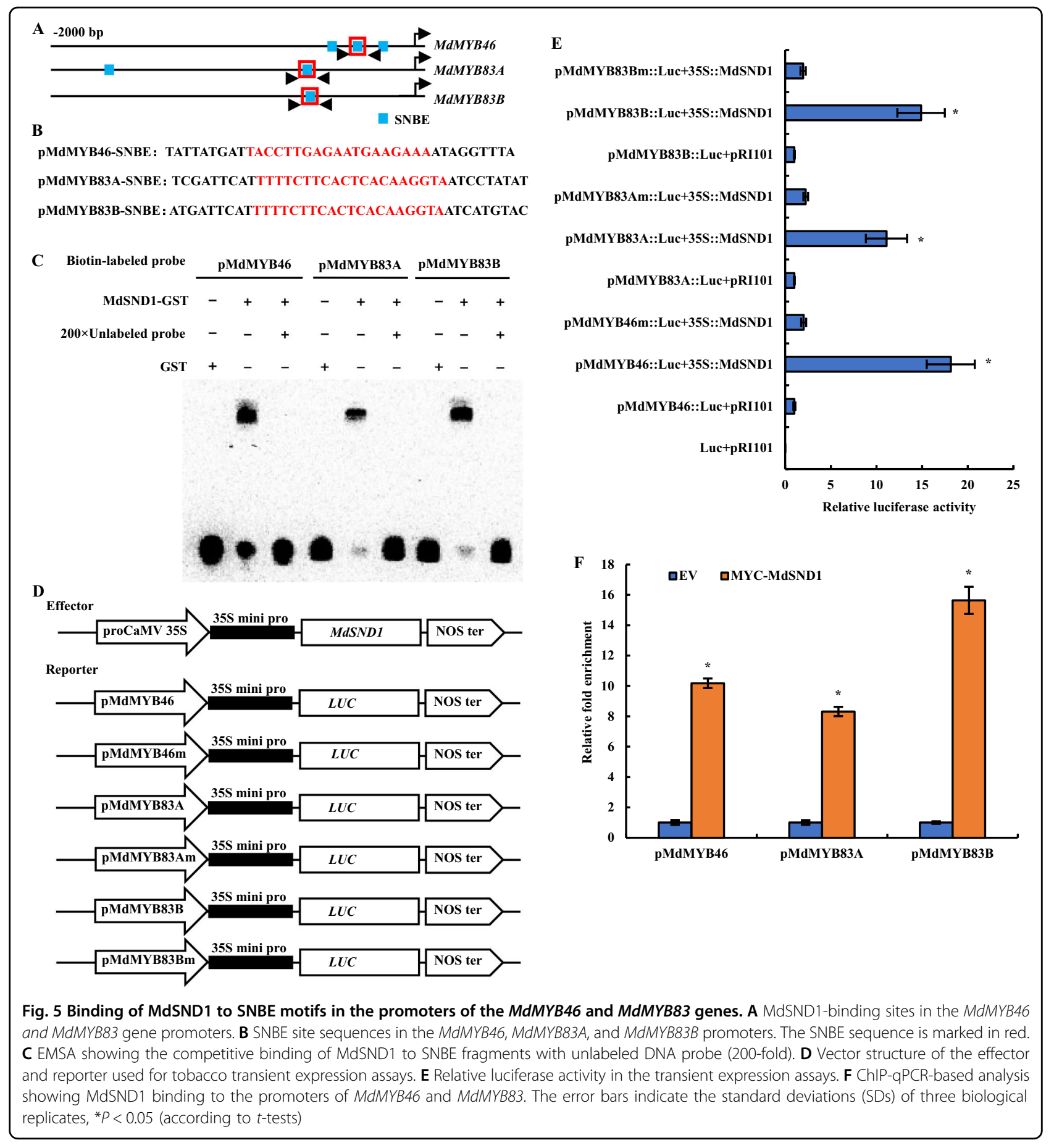

a mobility shift (Fig. 5C). However, mobility shifts were not seen when the 200-fold unlabeled MdMYB46/ $M d M Y B 83$ promoter fragments competed with the biotin-labeled promoter fragments and when the $M d M Y B 46 / M d M Y B 83$ promoter fragment was incubated with GST alone (Fig. 5C), indicating that the binding of MdSND1 to the MdMYB46 and MdMYB83 promoters was specific.
In addition, fragments (approximately 200-500 bp) of the promoters of the $M d M Y B 46, M d M Y B 83 A$, and $M d M Y B 83 B$ genes containing SNBE sites and the mutated SNBE sites (shown by the black arrow in Fig. 5D) were integrated into a luciferase reporter vector. p35S:: MdSND1 was then injected together with these reporters as an effector, and the luciferase activity in tobacco leaves was evaluated after $48 \mathrm{~h}$. The above results indicated that 
MdSND1 could activate transcription of the MdMYB46 and MdMYB83 genes (Fig. 5E).

To further verify the above results, a chromatin immunoprecipitation test was used to examine whether MdSND1 could bind to the promoters of MdMYB46 and MdMYB83 in vivo. We first overexpressed MYC-tagged SND1 in Ourin apple calluses. An anti-MYC antibody was then used to immunoprecipitate chromatin from MYCSND1 overexpressors cross-linked by formaldehyde for enrichment of MYC-SND1-bound DNA fragments. Finally, we used the immunoprecipitated DNA fragments as templates for qRT-PCR-based detection of $M d M Y B 83$ and MdMYB46 promoter sequences. The results showed that the fragments in the promoters of MdMYB46, $M d M Y B 83 A$, and $M d M Y B 83 B$ contained SNBE sites, which could be strongly bound by MdSND1 (Fig. 5F).

From the in vitro and in vivo binding analyses, we can conclude that MdSND1 directly binds to the promoters of the MYB46 and MYB83 genes to regulate their expression. In addition, we also analyzed the promoters of several lignin biosynthetic genes (MdCCR, MdCOMT, $M d H C T, M d F 5 H, M d 4 C L, M d C A D, M d C 3 H$, and $M d C 4 H$ ) (Fig. S4) and found that there were multiple SNBE sites in these genes (except $M d C 4 H$ ), which indicated that these genes might also be directly regulated by MdSND1.

\section{Expression of some stress-responsive genes is induced by MdSND1 in apple}

As shown in Fig. 2D, the expression of MdSND1 can be induced by $\mathrm{ABA}, \mathrm{NaCl}$, and mannitol. To determine the function of MdSND1 in the stress response, the relationships between MdSND1 and our prescreened stress response genes (MdAREB1A, MdAREB1B, MdDREB2A, $M d R D 22, M d R D 29 A$, and $M d R D 29 B)^{36}$ were investigated.

The transcript levels of these stress-responsive genes in MdSND1 transgenic and wild-type apple plants were measured. As shown in Fig. 6, the transcript levels of the MdAREB1A, MdAREB1B, MdDREB2A, MdRD22, and $M d R D 29 A$ genes were positively correlated with the level of SND1 in apple plants, but the transcript level of $M d R D 29 B$ changed statistically insignificantly in the overexpression and RNAi plants, indicating that the transcription of the MdAREB1A, MdAREB1B, $M d D R E B 2 A, M d R D 22$, and MdRD29A genes could be affected by MdSND1 in apple.

\section{MdSND1 activates the transcription of stress signaling genes by directly binding to SNBE motifs in their promoters}

The sequences of $2000 \mathrm{bp}$ of the promoters of $M d R D 29 A, M d A R E B 1 B, M d R D 22, M d A R E B 1 A$, and $M d D R E B 2 A$ were downloaded (https://iris.angers.inra.fr/ gddh13/jbrowse/?data $=$ gddh13) and analyzed, the results

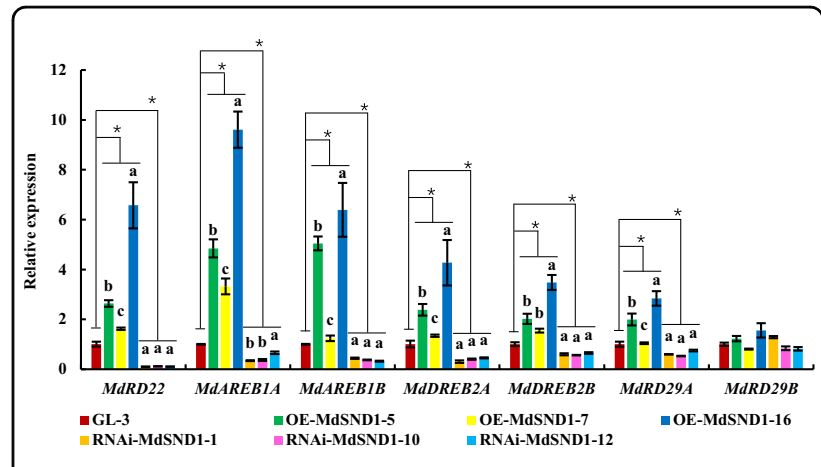

Fig. 6 Transcript levels of genes related to stress signaling in MdSND1 transgenic apple. The error bars indicate the standard deviations (SDs) of three biological replicates, ${ }^{*} P<0.05$ (according to $t$-tests). The letters indicate the level of significance $(P<0.05$, according to Duncan's multiple range test)

of which are shown in Fig. 7A. There were four SNBE sites in the promoter of $M d R D 22$, two SNBE sites in the promoter of $M d R D 29 A$, one SNBE site in the promoter of $M d A R E B 1$, three SNBE sites in the promoter of MdAR$E B 1 B$, and two SNBE sites in the promoter of $M d D R E B 2 A$, indicating that they all had the potential to be regulated by MdSND1.

To verify the interactions between MdSND1 and the SNBE binding sites in the promoters of MdAREB1A, $M d A R E B 1 B, M d D R E B 2 A, M d R D 22$, and MdRD29A, a ChIP approach was used to examine whether MdSND1 binds to these promoters in vivo. MYC-tagged SND1 was overexpressed in Ourin apple calli. After the steps of formaldehyde cross-linking chromatin and MYC antibody precipitating DNA fragments, we used the immunoprecipitated DNA fragments as templates for qRT-PCR for detection of MdRD29A, MdAREB1A, MdAREB1B, $M d R D 22$, and $M d D R E B 2 A$ promoter sequences. MdSND1 was found to bind to these sites to varying degrees (Fig. 7B).

The SNBE sequence (Fig. 7C) with the strongest binding ability among the MdAREB1A-P1, MdAREB1B-P3, MdDREB2A-P2, MdDR22-P3, and MdRD29A-P1 fragments was selected for designing biotin-labeled probes based on the results of the ChIP-qPCR. These probes were then incubated with GST-MdSND1 proteins. We used an electrophoretic mobility shift assay to detect binding between MdSND1 and the promoter fragments and an unlabeled probe (200-fold) for a competition assay. Fig. 7D shows that the promoters of the MdAREB1A, $M d A R E B 1 B, M d D R E B 2 A, M d R D 22$, and MdRD29A genes were bound to the MdSND1 protein.

We also inserted the fragments from the promoters of MdAREB1A-P1, MdAREB1B-P3, MdDREB2A-P2, $M d D R 22-P 3$, and MdRD29A-P1 and the corresponding mutated SNBE binding site sequences into a luciferasecontaining reporter gene vector for further verification 


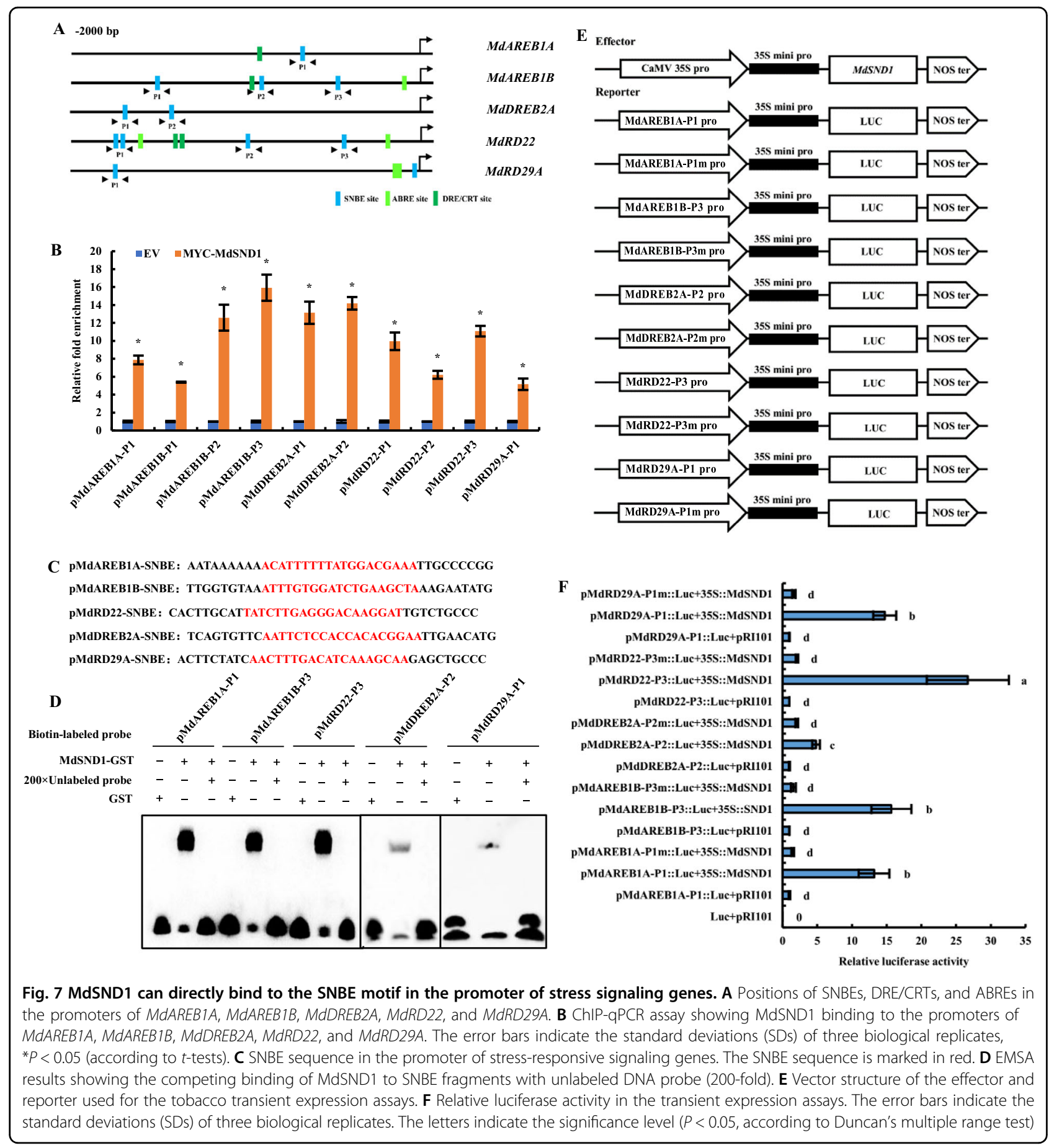

tests (Fig. 7E). The reporter vector was inserted into the leaves of tobacco together with either the effector p35S:: MdSND1 or a pRI101AN empty vector. The fluorescence distribution and luciferase activity results showed that MdSND1 could directly activate the expression of the MdAREB1A, MdAREB1B, MdDREB2A, MdRD22, and $M d R D 29 A$ genes by binding to their promoters.

\section{Discussion}

In Arabidopsis woody tissue, the key regulators of secondary wall formation are NST1 and SND1 (also known as NST3). SND1 and NST1 are functionally redundant in regulating secondary wall biosynthesis ${ }^{10,14}$, and the secondary wall thickening of interfibers and secondary xylem is completely inhibited in mutants with mutations in both of these 
genes ${ }^{10}$. AtSND1 has a dual role in plant growth: it either directly binds to the MYB46 promoter region, thus activating the lignin biosynthesis pathway, or directly binds to the ABI4 gene promoter, thereby maintaining low levels of $\mathrm{ABA}$ and inhibiting growth ${ }^{13}$. In the present study, MdSND1 and AtSND1 showed some functional similarities in regulating lignin synthesis. The lignin content increased in apple plants overexpressing MdSND1 but decreased in RNAi plants (Fig. S3). MdSND1 was able to activate the transcription of $M d M Y B 46 / 83$ and indirectly regulate the accumulation of lignin in apple. However, whether SND1 can function like MdMYB46 and directly act on the promoter of lignin biosynthesis genes remains unknown ${ }^{36}$.

Both drought and salt stress can lead to changes in osmotic pressure in cells, while salt also has additional ionic or iontoxicity effects ${ }^{1,38}$. Elucidating the signal transduction response pathway involved in both salt and osmotic stress is of great significance for clarifying the antistress mechanism of plants. This knowledge is essential for guiding the development of varieties with broad adaptability. In the present study, the transcription of $M d S N D 1$ was induced by abiotic stress (Fig. 2D). Moreover, the MdSND1-overexpressing plants showed higher salt and osmotic stress tolerance than did the nontransgenic plants, while the MdSND1-RNAi plants were more vulnerable, similar to the AtSND1 deletion mutant seedlings, indicating that the transcript level of $M d S N D 1$ is intrinsically linked to the stress tolerance of apple. A series of stress-responsive pathways in plants is triggered under salt and osmotic stress ${ }^{1,20}$, in which both ABA-dependent and ABA-independent regulatory systems are involved ${ }^{2}$. The bZIP TF AREB and the DREB AP2/ERF TF family members have pivotal functions in ABAdependent and ABA-independent gene expression, respectively $^{39-42}$. In addition, several stress-responsive genes, such as $R D 22$ and $R D 29 A$, are also activated by dehydration, high salinity, and cold stress and function in signal transduction pathways $^{38,43}$. In apple, MdRD29A, MdRD29B, MdAREB3.2 (MdAREB1A), MdAREB2 (MdAREB1B), MdDREB2A, $M d D R E B 2 B$, and $M d R D 22$ were recently found to be responsive to stress signals ${ }^{44-47}$. In this study, the transcript levels of MdRD22, MdAREB1A, MdRD29A, MdAREB1B, and MdDREB2A were up- or downregulated in MdSND1overexpressing or -RNAi apple plants, respectively (Fig. 6), and ChIP assays, EMSAs and luciferase reporter transcription activity assays further confirmed that MdSND1 could bind to the SNBE motifs in the promoters of the five genes and activate their expression (Fig. 7), which indicates that MdSND1 could have a role in activation of stress signal transduction pathways (Fig. 8).

SND1 belongs to the NAC superfamily, which is one of the largest plant-specific transcription factor families ${ }^{48}$. NAC TFs regulate the transcription of downstream target genes by binding to multiple motifs, such as NAC-binding site $(N A C B S s)$, in which GCTT is the core binding motif ${ }^{49,50}$, the

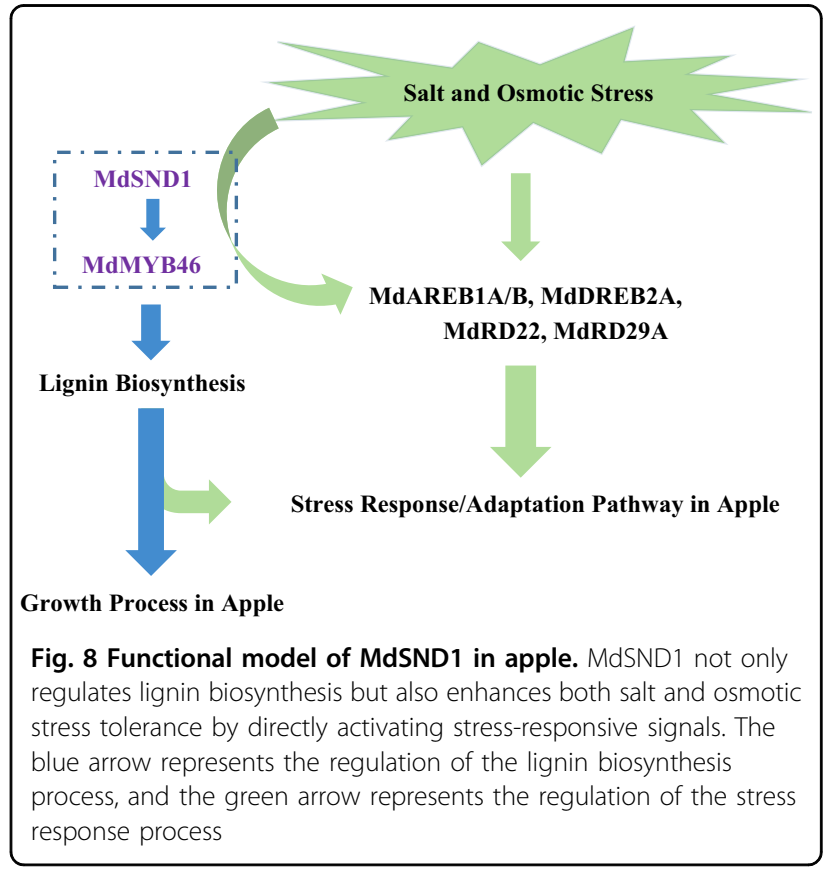

core sequence $\mathrm{CA}(\mathrm{A} / \mathrm{C}) \mathrm{G}(\mathrm{T} / \mathrm{C})(\mathrm{T} / \mathrm{C} / \mathrm{A})(\mathrm{T} / \mathrm{C} / \mathrm{A})^{51}$ and SNBEs, in which (T/A)NN(C/T) (T/C/G)TNNNNNNNA $(\mathrm{A} / \mathrm{C}) \mathrm{GN}(\mathrm{A} / \mathrm{C} / \mathrm{T})(\mathrm{A} / \mathrm{T})$ is the consensus sequence ${ }^{52}$. Several NAC transcription factors in different plant species have been shown to be closely correlated with the stress-related signal transduction pathway and with abiotic stress tolerance $^{15,18,48}$. In Arabidopsis thaliana, by binding to the TTGCGTA motif in its promoter, ATAF1 regulates the transcription of NCED3, which encodes the key enzyme in ABA biosynthesis under dehydration stress ${ }^{53,54}$. AtANAC016 has been reported to be a positive regulator of plant tolerance to drought stress $^{55}$. In apple, salt stress induces the expression of MdNAC047, which promotes the accumulation of ethylene by activating the expression of MdERF3, modulating plant salt tolerance ${ }^{45}$. In our study, MdSND1 could activate the transcription of stress-responsive genes by directly binding to SNBE sites, which reaffirms the important role of NAC regulators in regulating the stress signal transduction pathway.

\section{Conclusions}

In this study, the key NAC transcription factor SND1, which is involved in the lignin biosynthesis process, was functionally analyzed in apple. MdSND1 could promote the accumulation of lignin and enhanced both salt and osmotic stress tolerance in apple. MdSND1 regulates the biosynthesis of lignin by activating the transcription of MdMYB46/ 83 and participates in the stress signal transduction pathway by directly activating stress-response signals (MdAREB1A, MdAREB1B, MdDREB2A, MdRD29A, and MdRD22). 


\section{Methods}

\section{Plant materials}

Tissue culture-generated GL-3 apple plants were selected from Malus $\times$ domestica $\mathrm{cv}$. Royal Gala seedlings in our laboratory ${ }^{56}$. GL-3 plantlets were subcultured in medium (MS medium supplemented with $0.3 \mathrm{mg} / \mathrm{L} 6-\mathrm{BA}$, $0.2 \mathrm{mg} / \mathrm{L}$ IAA and $0.1 \mathrm{mg} / \mathrm{L} \mathrm{GA}_{3}$ ) under long-day conditions (14 h light:10 h dark) at $25^{\circ} \mathrm{C}$.

\section{Vectors and transformation}

The DNA sequence of MdSND1 in GL-3 was the same as that of MD06G1121400 in the apple genome (https://iris. angers.inra.fr/gddh13/jbrowse/?data=gddh13). MdSND1 overexpression and RNAi vectors were constructed for apple transformation according to the methods of Chen et al. ${ }^{36}$. The overexpression and RNAi vectors of MdSND1 were then introduced into Agrobacterium tumefaciens strain EHA105 for GL-3 transformation ${ }^{56}$. The primers used are listed in Table S1.

The pRI-MYC-MdSND1 vector for callus transformation was constructed on the basis of the pRI-MdSND1 vector, with the MYC tag added before the MdSND1 fulllength CDS. The fusion vector was then introduced into EHA105 for Ourin callus transformation ${ }^{56}$, after which a successfully transformed callus was used for subsequent chromatin immunoprecipitation.

Stress treatments; measurements of lignin, proline, $\mathrm{H}_{2} \mathrm{O}_{2}$, MDA and relative water content; and NBT/DAB staining

The following were performed as described in ref. ${ }^{36}$ : short/long-term stress treatments; lignin, proline, $\mathrm{H}_{2} \mathrm{O}_{2}$, $\mathrm{MDA}$ and relative water content measurements; and NBT/DAB staining.

\section{ChIP assays}

A 20-day-old positive callus transformed with pRI-MYCMdSND1 was screened five times before being used for chromatin immunoprecipitation experiments via a ChIPqPCR kit (Farmingdale, USA) according to the manufacturer's instructions ${ }^{36}$. For ChIP-quantitative PCR analysis, MdACTIN was used as an endogenous control for normalization, and the fold enrichment of MdSND1-bound DNA fragments was calculated by comparing the samples with the inputs. All the primers used are listed in Table S1.

\section{EMSAs}

The full-length MdSND1 CDS region fused to a GST tag was transformed into Escherichia coli BL21 to produce a GST-MdSND1 recombinant protein, which was further purified and incubated together with SNBE oligonucleotides marked with biotin-11-UTP-labeled DNA fragments from the promoters of osmotic stress-responsive genes (MdAREB1A, MdAREB1B, MdDREB2A, MdRD22, and MdRD29A) for $30 \mathrm{~min}$. The DNA signals were detected by chemiluminescence (Biyuntian, Shanghai, China). For the competition assays, unlabeled oligonucleotides (labeled probes at 200-fold) were added to the above binding buffer (Biyuntian).

\section{Analysis of transcription activity}

The 200-500 bp fragments containing the corresponding binding sites in the promoters of $M d A R E B 1 A, M d A R E B 1 B$, $M d D R E B 2 A, M d R D 22$, and $M d R D 29 A$ were cloned and inserted into a pRI-mini35S-LUC vector. The newly constructed pRI-mini35S-LUC vector was used as a reporter; the pRI-MdSND1 vector served as an effector; and pRI-mini35SLUC vectors containing mutated conserved binding sites from the $M d A R E B 1 A, M d A R E B 1 B, M d D R E B 2 A, M d R D 22$, and $M d R D 29 A$ promoter fragments were used as controls. The transcription activation assay was performed according to a previously described method ${ }^{36}$.

\section{RNA extraction and qRT-PCR}

The methods of RNA extraction and qRT-PCR were the same as previously described ones ${ }^{36}$.

\section{Acknowledgements}

We thank Dr. Kekun Zhang and AJE for their help with language editing. This work was supported by grants from the National Natural Science Foundation of China (31972380; 31170635). The funding body had no role in the design of the study or in the collection, analysis, and interpretation of data or in the writing of the manuscript.

\section{Author contributions}

H.D. and Z.Z. conceived the research plan and supervised the experiments; K.C. Y.G., and M.S. performed most of the experiments and analyzed the data; L.L. and H.X. assisted in the management of the transgenic plants; and K.C. wrote the article. All of the authors read and approved the manuscript.

\section{Availability of data and materials}

The datasets used and/or analyzed during the current study are available from the corresponding author upon reasonable request. The genes sequences can

downloaded from the NCBI database, and the genes and their accession numbers are as follows: MdMYB46 (XM_008365407.3), MdMYB83B (XM_008346410.3), MdMYB83A (XM_008376953.3), MdMYB63 (XM_008384900.3), MdMYB58 (XM_008373948.3), MdDREB2B (MG099825.1), MdDREB2A (XM_008355947.3), MdAREB1B (XM_008374912.3), MdAREB1A (XM_029094247.1), MdRD29B (XM_008378353.3), MdRD29A (XM_008345499.3), MdRD22 (XM_017333810.2), MdCCR (XM_008377320.3), MdCOMT (XM_008387517.3), Md4CL (XM_029091143.1), MdHCT (XM_008344601.3), MdF5H (XM_008339691.3), MdC3H (XM_008380681.3), MdC4H (NM_001294106.1), PtrWND1A (HQ215847), PtrWND1B (HQ215848), PtrWND2A (HQ215849), PtrWND2B (HQ215850), PtrWND3A (HQ215851), PtrWND3B (HQ215852), PtrWND4A (HQ215853), PtrWND4B (HQ215854), PtrWND5A

(HQ215855), PtrWND5B (HQ215856), PtrWND6A (HQ215857), PtrWND6B (HQ215858), PtrNAC054 (XM_002316127), PtrNAC058 (XM_002311240), PtrNAC064 (XM_002319836), and PtrNAC066 (XM_002329142), PtrNAC154 (XM_002327995), PtrNAC156 (XM_002309731), PtrNAC105 (XM_002329769), EgWND1 (HQ215846), MtNST1 (GU144511), OsSWN1 (JN634070), OsSWN2 (JN634071), OsSWN3 (JN634072), OsSWN4 (JN634073), OsSWN5 (JN634074), OsSWN6 (JN634075), OsSWN7 (JN634076), OsSWN8 (XM_006647538), OsSWN9 (XM_006658959), BdSWN1 (JQ693422), BdSWN2 (JQ693423), BdSWN3 (JQ693424), BdSWN4 (JQ693425), BdSWN5 (JQ693426), BdSWN6 (JQ693427), BdSWN7 (JQ693428), BdSWN7 (JQ693429), ZmSWN1 (JN634077), ZmSWN2 (JN634078), ZmSWN3 (JN634079), ZmSWN4 (JN634080), ZmSWN5 (JN634081), ZmSWN6 (JN634082), and ZmSWN7 (JN634083).

Conflict of interest

The authors declare that they have no conflict of interest. 
Supplementary Information accompanies this paper at (https://doi.org/ 10.1038/s41438-020-00433-7).

Received: 24 May 2020 Revised: 8 September 2020 Accepted: 17 October 2020

Published online: 01 December 2020

\section{References}

1. Zhu, J. K. Abiotic stress signaling and responses in plants. Cell 167, 313-324 (2016).

2. Yamaguchi-Shinozaki, K. \& Shinozaki, K. Organization of cis-acting regulatory elements in osmotic-and cold-stress-responsive promoters. Trends Plant Sci. 10, 88-94 (2005).

3. Yoshida, T., Mogami, J. \& Yamaguchi-Shinozaki, K. ABA-dependent and ABAindependent signaling in response to osmotic stress in plants. Curr. Opin. Plant Biol. 21, 133-139 (2014).

4. Xie, Q. Arabidopsis NAC1 transduces auxin signal downstream of TIR1 to promote lateral root development. Gene. Dev. 14, 3024-3036 (2000).

5. Duval, M., Hsieh, T. F., Kim, S. Y. \& Thomas, T. L. Molecular characterization of AtNAM: a member of the Arabidopsis NAC domain superfamily. Plant Mol. Biol. 50, 237-248 (2002).

6. Ernst, H. A., Nina Olsen, A., Skriver, K., Larsen, S. \& Lo-Leggio, L. Structure of the conserved domain of ANAC, a member of the NAC family of transcription factors. EMBO Rep. 5, 297-303 (2004).

7. $\mathrm{Hu}, \mathrm{H}$. et al. Overexpressing a NAM, ATAF, and CUC (NAC) transcription factor enhances drought resistance and salt tolerance in rice. Proc. Natl Acad. Sci. USA 103, 12987-12992 (2006).

8. Souer, E., van Houwelingen, A., Kloos, D., Mol, J. \& Koes, R. The no apical meristem gene of Petunia is required for pattern formation in embryos and flowers and is expressed at meristem and primordia boundaries. Cell $\mathbf{8 5}$ 159-170 (1996).

9. Zhong, R., Demura, T. \& Ye, Z. H. SND1, a NAC domain transcription factor, is a key regulator of secondary wall synthesis in fibers of Arabidopsis. Plant Cell 18, 3158-3170 (2006).

10. Zhong, R., Richardson, E., Ye, Z. H. \& Two, N. A. C. domain transcription factors, SND1 and NST1, function redundantly in regulation of secondary wall synthesis in fibers of Arabidopsis. Planta 225, 1603-1611 (2007).

11. Yang, S. D., Seo, P. J., Yoon, H. K. \& Park, C. M. The Arabidopsis NAC transcription factor VNI2 integrates abscisic acid signals into leaf senescence via the COR/RD genes. Plant Cell 23, 2155-2168 (2011).

12. Zhong, R. \& Ye, Z. H. Transcriptional regulation of lignin biosynthesis. Plant Signal. Behav. 4, 1028-1034 (2009).

13. Jeong, C. Y. et al. Dual role of SND1 facilitates efficient communication between abiotic stress signalling and normal growth in Arabidopsis. Sci. Rep. 8 , 10114 (2018).

14. Mitsuda, N. et al. NAC transcription factors, NST1 and NST3, are key regulators of the formation of secondary walls in woody tissues of Arabidopsis. Plant Cell 19, 270-280 (2007).

15. Nakashima, K., Takasaki, H., Mizoi, J., Shinozaki, K. \& Yamaguchi-Shinozaki, K. NAC transcription factors in plant abiotic stress responses. Biochim. Biophys. Acta 1819, 97-103 (2012).

16. Sun, L., Li, D., Zhang, H. \& Song, F. Functions of NAC transcription factors in biotic and abiotic stress responses in plants. Hereditas (Beijing) 34, 993-1002 (2012).

17. Ramegowda, V. et al. Expression of a finger millet transcription factor, EcNAC1, in tobacco confers abiotic stress-tolerance. PLoS ONE 7, e40397 (2012).

18. Nuruzzaman, M., Sharoni, A. M. \& Kikuchi, S. Roles of NAC transcription factors in the regulation of biotic and abiotic stress responses in plants. Front. Microbiol. 4, 1-16 (2013).

19. Zhu, J. K. Plant salt tolerance. Trends Plant Sci. 6, 66-71 (2001).

20. Zhu, J. K. Salt and drought stress signal transduction in plants. Annu. Rev. Plant Biol. 53, 247-273 (2002).

21. Ouyang, B. et al. Identification of early salt stress response genes in tomato root by suppression subtractive hybridization and microarray analysis. J. Exp. Bot. 58, 507-520 (2007).

22. Li, D., Su, Z., Dong, J. \& Wang, T. An expression database for roots of the model legume Medicago truncatula under salt stress. BMC Genomics 10, 517 (2009).
23. Yadav, N. R., Taunk, J., Rani, A., Aneja, B. \& Yadav, R. C. in Climate Change and Plant Abiotic Stress Tolerance (eds Narendra T. \& Sarvajeet S. G.) 605-640 (Wiley Blackwell, 2013).

24. Agarwal, P. K., Agarwal, P., Reddy, M. K. \& Sopory, S. K. Role of DREB transcription factors in abiotic and biotic stress tolerance in plants. Plant Cell Rep. 25, 1263-1274 (2006).

25. Shen, $\mathrm{H}$. et al. OsWRKY30 is activated by MAP kinases to confer drought tolerance in rice. Plant Mol. Biol. 80, 241-253 (2012).

26. Nakashima, K. et al. Organization and expression of two Arabidopsis DREB2 genes encoding DRE-binding proteins involved in dehydration- and highsalinity-responsive gene expression. Plant Mol. Biol. 42, 657-665 (2000).

27. Lee, S. J. et al. DREB2C interacts with ABF2, a bZIP protein regulating abscisic acid responsive gene expression, and its overexpression affects abscisic acid sensitivity. Plant Physiol. 153, 716-727 (2010).

28. Chen, M. et al. GmDREB2, a soybean DRE-binding transcription factor, conferred drought and high-salt tolerance in transgenic plants. Biochem. Bioph. Res. Commun. 353, 299-305 (2007).

29. Chen, J., Xia, X. \& Yin, W. Expression profiling and functional characterization of a DREB2-type gene from Populus euphratica. Biochem. Bioph. Res. Commun. 378, 483-487 (2009).

30. Cui, M. et al. Induced over-expression of the transcription factor OsDREB2A improves drought tolerance in rice. Plant Physiol. Biochem. 49, 1384-1391 (2011).

31. Li, X. et al. EsDREB2B, a novel truncated DREB2-type transcription factor in the desert legume Eremosparton songoricum, enhances tolerance to multiple abiotic stresses in yeast and transgenic tobacco. BMC Plant Biol. 14, 44 (2014).

32. Hichri, I. et al. SIDREB2, a tomato dehydration-responsive element-binding 2 transcription factor, mediates salt stress tolerance in tomato and Arabidopsis. Plant Cell Environ. 39, 62-79 (2015).

33. Yoshida, T. et al. Four Arabidopsis AREB/ABF transcription factors function predominantly in gene expression downstream of SnRK2 kinases in abscisic acid signalling in response to osmotic stress. Plant Cell Environ. 38, 35-49 (2015).

34. Narusaka, Y. et al. Interaction between two cis-acting elements, ABRE and DRE, in ABA-dependent expression of Arabidopsis rd29A gene in response to dehydration and high-salinity stresses. Plant J. 34, 137-148 (2003).

35. Yamaguchi-Shinozaki, K. \& Shinozaki, K. Transcriptional regulatory networks in cellular responses and tolerance to dehydration and cold stresses. Annu. Rev. Plant Biol. 57, 781-803 (2006).

36. Chen, K. et al. MdMYB46 could enhance salt and osmotic stress tolerance in apple by directly activating stress responsive signals. Plant Biotechnol. J. 17, 2341-2355 (2019).

37. McCarthy, R. L., Zhong, R. \& Ye, Z. H. MYB83 is a direct target of SND1 and acts redundantly with MYB46 in the regulation of secondary cell wall biosynthesis in Arabidopsis. Plant Cell Physiol. 50, 1950-1964 (2009).

38. Shi, H., Lee, B. H., Wu, S. J. \& Zhu, J. K. Overexpression of a plasma membrane $\mathrm{Na}^{+} / \mathrm{H}^{+}$antiporter gene improves salt tolerance in Arabidopsis thaliana. Nat. Biotechnol. 21, 81-85 (2003).

39. Yamaguchi-Shinozaki, K. \& Shinozaki, K. A novel cis-acting element in an Arabidopsis gene is involved in responsiveness to drought, low-temperature, or high-salt stress. Plant Cell 6, 251-264 (1994).

40. Abe, $\mathrm{H}$. et al. Arabidopsis AtMYC2 (bHLH) and AtMYB2 (MYB) function as transcriptional activators in abscisic acid signaling. Plant Cell 15, 63-78 (2003).

41. Thomashow, M. F. Molecular basis of plant cold acclimation: insights gained from studying the CBF cold response pathway. Plant Physiol. 154, 571-577 (2010).

42. Lata, C. \& Prasad, M. Role of DREBs in regulation of abiotic stress responses in plants. J. Exp. Bot. 62, 4731-4748 (2011).

43. Yamaguchi-Shinozaki, K. \& Shinozaki, K. The plant hormone abscisic acid mediates the drought-induced expression but not the seed-specific expression of $r d 22$, a gene responsive to dehydration stress in Arabidopsis thaliana. Mol. Gen. Genet. 238, 17-25 (1993).

44. Zhao, T., Liang, D., Wang, P., Liu, J. \& Ma, F. Genome-wide analysis and expression profiling of the DREB transcription factor gene family in Malus under abiotic stress. Mol. Genet. Genomics 287, 423-436 (2012).

45. Ma, Q. et al. An apple CIPK protein kinase targets a novel residue of AREB transcription factor for ABA-dependent phosphorylation. Plant Cell Environ. 40, 2207-2219 (2017)

46. An, J. P. et al. An apple NAC transcription factor enhances salt stress tolerance by modulating the ethylene response. Physiol. Plant. 164, 279-289 (2018).

47. Shao, Y., Zhang, X., van Nocker, S., Gong, X. \& Ma, F. Overexpression of a protein kinase gene MpSnRK2.10 from Malus prunifolia confers tolerance to drought stress in transgenic Arabidopsis thaliana and apple. Gene 692, 26-34 (2019). 
48. Shao, H., Wang, H. \& Tang, X. NAC transcription factors in plant multiple abiotic stress responses: progress and prospects. Front. Plant Sci. 6, 902 (2015).

49. Simpson, S. D. et al. Two different novel cis-acting elements of erd1, a clpA homologous Arabidopsis gene function in induction by dehydration stress and dark-induced senescence. Plant J. 33, 259-270 (2003).

50. Kim, S. G., Kim, S. Y. \& Park, C. M. A membrane-associated NAC transcription factor regulates salt-responsive flowering via FLOWERING LOCUS T in Arabidopsis. Planta 226, 647-654 (2007).

51. Ogo, Y. et al. A novel NAC transcription factor, IDEF2, that recognizes the iron deficiency-responsive element 2 regulates the genes involved in iron homeostasis in plants. J. Biol. Chem. 283, 13407-13417 (2008).

52. Zhong, R., Lee, C. \& Ye, Z. H. Global analysis of direct targets of secondary wall NAC master switches in Arabidopsis. Mol. Plant 3, 1087-1103 (2010).
53. Jensen, M. K. et al. Transcriptional regulation by an NAC (NAM-ATAF1,2-CUC2) transcription factor attenuates ABA signalling for efficient basal defence towards Blumeria graminis f. sp. hordei in Arabidopsis. Plant J. 56, 867-880 (2008).

54. Jensen, J. K., Johnson, N. \& Wilkerson, C. G. Discovery of diversity in xylan biosynthetic genes by transcriptional profiling of a heteroxylan containing mucilaginous tissue. Front. Plant Sci. 4, 183 (2013).

55. Sakuraba, Y., Kim, Y. S., Han, S. H., Lee, B. D. \& Paek, N. C. The Arabidopsis transcription factor NAC016 promotes drought stress responses by repressing AREB1 transcription through a trifurcate feed-forward regulatory loop involving NAP. Plant Cell 27, 1771-1787 (2015).

56. Dai, H. et al. Development of a seedling clone with high regeneration capacity and susceptibility to Agrobacterium in apple. Sci. Hortic. 164, 202-208 (2013). 